Journal of Engineering and Applied Sciences 14 (1): 83-87, 2019

ISSN: 1816-949X

(C) Medwell Journals, 2019

\title{
Patient Monitoring and Tracking by Using Wireless Sensor Network
}

\author{
Arshad Ali \\ Faculty of Computer and Information Systems, The Islamic University of Madinah, \\ Prince Naif Ibn Abdulaziz Road, 42351 Al Jamiah Al Madinah Al Munawarah, Saudi Arabia
}

\begin{abstract}
Aim of the proposed research work is to monitoring and rescues the patient who are in critical condition. The proposed system will be capable to monitor human body temperature, blood pressure and heart rate. The sensed information will be sent to the cross ponding base station where it will be analysed and the decision will be made by expert. The expert decision will depend on the medical history of the patient and then rescue team will be instructed to rescue the cross ponding patient. In case of the heart patient the information will be very critical as the proposed system will be capable to detect condition of the patient well before the heart attack. In the first phase the proposed algorithm is implemented theoretical by using matlab and in the second phase, the algorithm will be implemented practically by creating prototype hardware along with all the modules.
\end{abstract}

Key words: Sensor, blood, pressure, heart, beat, implemented

\section{INTRODUCTION}

WSN's are very promising for monitoring extraordinary diverse environments. A WSN consists of wireless nodes that are able to sense some physical information, process and transfer to the destination by establishing wireless ad-hoc networks. A WSN consists of tiny sensor nodes each capable of sensing some phenomenon, process data and communicate with its neighbours (Cressie, 1993).

The use of WSN dramatically increased in electrical and electronic equipment in the field of medical research and clinical purposes in the last quarter of a century. In medical devices, the main function is to measure or determine the presence of a physical quantity that can be useful for diagnostic purposes. Therefore, many types of instrumentation systems are used in hospitals and physician's clinics. The fundamental purpose of medical instrumentation is to measure or determine the presence of some physical phenomena that may assist the medical personnel to make better diagnosis and treatment. Therefore, so many types of equipment is used in the medical facilities and hospitals. In the broadest sense, any medical instrument would comprise of the following four basic functional components: measure and Transducer/Sensor, signal conditioner and display system. Patient monitoring system in all ICUs is the most needed and essential device for monitoring the patient's vitals (Subudhi and Sivanandam, 2014).

Recent technological advances in integrated digital electronics and miniaturization of physical sensors, microprocessor and radio frequency devices into a single microchip has led to the emergence of very light weight, ultra-low power, monitoring sensor devices (Alumona et al., 2014). These sensor devices have the capability of sensing, processing and transmitting vital physiological signals using wireless technology (Varshney, 2005). Contrary to the traditional sensor networks that are carefully planned and deployed in the predetermined positions, WSNs can be deployed in an ad-hoc manner which make them robust, fault tolerance and increase in spatial coverage. They can greatly be used to monitor and track conditions of patients in both cities and rural areas using an intranet or internet thereby reducing the stress and strain of healthcare providers, eliminate medical errors, reduce workload, increase efficiency of hospital staff, reduce long-term cost of healthcare services and improve the comfort of the patients (Vijendra, 2011).

This study, proposes a networking solution in which sensor (Medical Super Sensor-MSS) is used to collect multiple physiological signs sensed by each of the body sensors in WBAN and forward them to the personal server. An Intelligent Personal Digital Assistant (IPDA) is used as a personal server, it has the ability to collect patient's vital signs and prioritizes the data transmission based on patient's current condition and data content.

Literature review: In recent years Wireless Sensor Networks (WSN's) have strongly emerged in the medical field for healthcare of the patient for data collection regarding the physical, psychological, cognitive and behavioural processes for monitoring purposes. During 


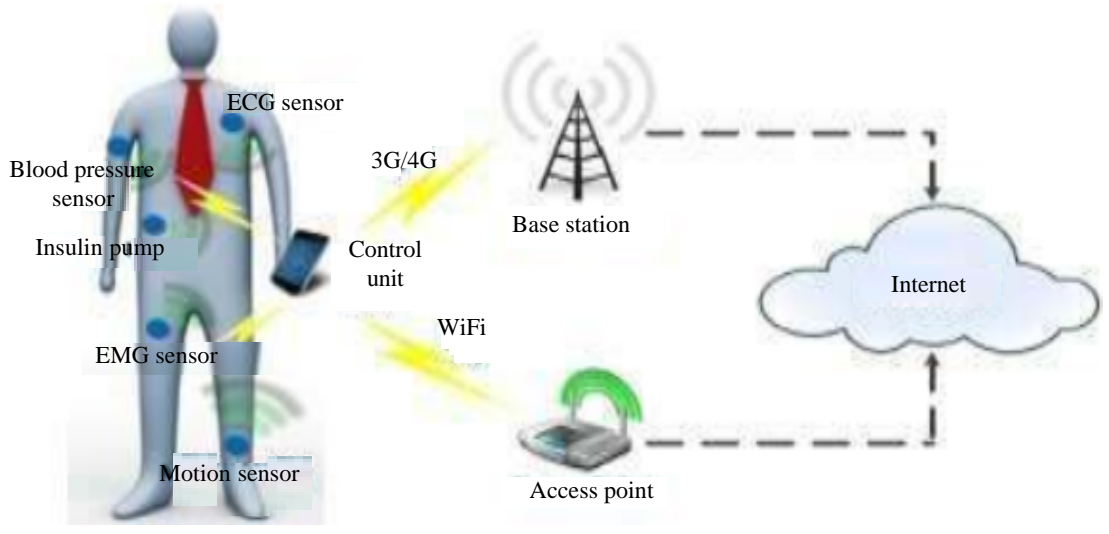

Fig. 1: Body area and communication network (Lai et al., 2009)

the literature review, we studied and representing some applications in the healthcare. Hook application are directed towards monitoring in mass-casualty disasters, while triage protocols for emergency medical services already exist (Hodgetts and Mackaway, 1995; Super et al., 1994), their effectiveness can quickly degrade with increasing number of victims. However, there is a need to improve the assessment of the first responder's health status during such mass-casualty disasters. The increased portability, scalability and rapidly deployable nature of wireless sensing systems can be used to automatically report the triage levels of numerous victims and continuously track the health status of first responders at the disaster scene more effectively (Super et al., 1994; Hodgetts and Mackaway, 1995). Diseases such as high blood pressure, asthma, diabetes, chronic obstructive pulmonary disease, congestive heart failure and memory decline are challenging to monitor and treat. These diseases can benefit from patients taking an active role in the monitoring process. Wirelessly networked sensors embedded in people's living spaces or carried on the person can collect information about personal physical, physiological and behavioural states and patterns in real-time and everywhere. Such data can also be correlated with social and environmental context. The researchers of Othman (2006) and Jamaluddin (2008) adopted PPG in their design. The researchers (Varshney, 2005) presented their idea of a device under development to monitor the cardiovascular status of patients. It is used to monitor the cardiovascular status of patients. It stores all recorded physiological signals (ECG, PPG photo plethysmograph) for medical post-processing. They also suggested that the family doctor may be notified by a text message sent automatically to the case of the critical value of the parameter measured occurs. However, no other results of this research will be presented. A practical system for monitoring heart rate, he does not use the auto-counting of blood or ECG signal pulse is generated
(Othman, 2006). The sensor output is viewed by the number of hours of counting the counter display using the 7-segment display. An updated results in counter display for reading it every $2 \mathrm{sec}$. Heart rhythm method is adopted in the development of this project (Fig. 1).

\section{MATERIALS AND METHODS}

Objectives: The main objective of this research to monitor the critical patient condition in real time and also keep the track record of the medical history. Secondly, in the case of emergency it will be quick and easy to locate the patient and rescue via the emergency services for medical treatment.

Proposed system and units: The proposed algorithm would constantly monitor body parameters, e.g., heartbeat temperature and compare it against a predetermined value set and if these values cross a particular limit it would automatically alert the doctor by initiating emergency call. In such case the patient will get a veryquick medical help and also would save time. While proposing this algorithm the heart patient is considered as highest priority for data comparison and analysis. The heart patient who have the medical history like they have heart attack before the system will detect heart health based on the current and previous data, after data analysis the system automatically call the emergency service number and the affected person will be rescued before the some medical condition. The rescue will service will provide him the first aid on the spot and get will more time to survive. The proposed system will enables medical professionals to monitor a patient remotely using various technological devices. This method is primarily used for managing chronic diseases or specific conditions such as heart disease, diabetes or asthma. The block diagram of the proposed is give in Fig. 2. 
J. Eng. Applied Sci., 14 (1): 83-87, 2019

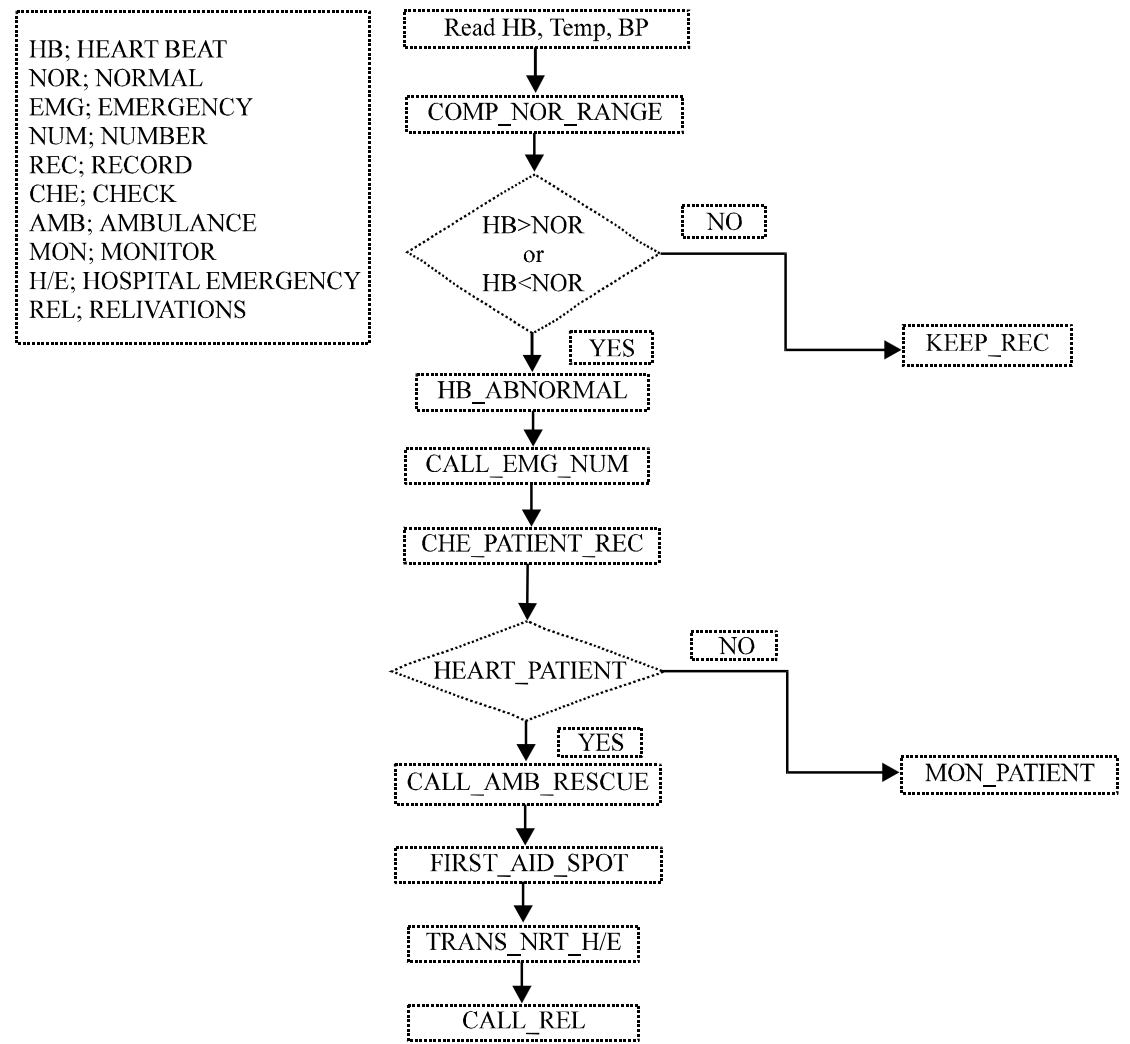

Fig. 2: Proposed algorithm for patient monitoring

Blood pressure sensor: Blood pressure sensor unit is used to measure the arterial blood pressure flowing through the blood vessels against the walls of the arteries. It measures the pressure signal caused by the interaction between the cuff and the blood flow through the brachial artery. The sensor produces an output voltage that varies in a linear way with pressure. Special circuitry minimizes the errors that might be caused by changes in temperature. If blood flow is normal, then blood pressure is normal (average 120/80). If blood flow becomes restricted in some way, blood pressure goes up. If increased blood pressure goes undetected, the person is at risk of severe medical problems.

Temperature sensor: This unit measures the temperature of patient and it also known as analog temperature sensor. The output voltage is linearly proportional to the temperature in celsius. It uses a solid state technology to determine the temperature. If the temperature increases means voltage also increases. By amplifying the voltage change it is easy to generate an analog signal which is directly proportional to temperature.

Heart beat rate: $A$ sensor unit is integrated in wrist band and it is weared by patient in left wrist and it measures the

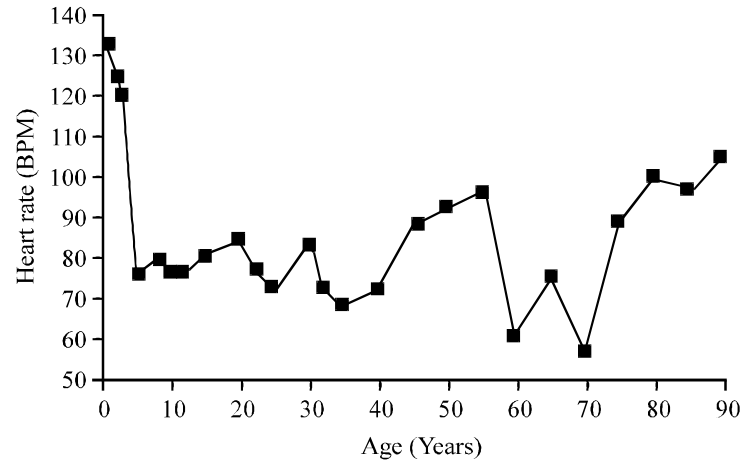

Fig. 3: Heartbeat according age group

heart beat by using the defined interval and send it to the central unit where it is processed. At central unit it is saved and compared with the standard and previous measurement if it finds any abnormality in the heart beat then it reported to concerned system and issues an alert Fig. 3.

GPS module: The Global Positioning System (GPS) module is a satellite navigation system that can be used to locate the positions anywhere on the globe. Designed and implemented by the Defense Department of the 
United States which includes satellites, control and monitoring stations and receivers. GPS receivers receive training transmitted by satellites and uses triangulation to calculate the user's exact GPS location. This unit is very important to rescue the patient by using the GPS, rescue services easily locate the affected person.

GSM module: This module has very important part in whole of the system it sends the message to emergency services/doctor regarding the patient assessment for the medical treatment. The GSM modem consists of a slot for inserting Subscriber Identity Module (SIM). GSM network generally contains three major stations. Mobile station, Base station subsystem and network subsystem. The mobile station contains an International Mobile station Equipment Identity (IMEI) number and SIM has IMSI number. The base station subsystem contains base transceiver station which has an antenna for communication and a base station controller that controls multiple base stations. Network subsystem contains Visitor Location Register (VLR), Home Location Register (HLR), Authentication Centre (AuC) and Equipment Identity Register (EIR).

Simulation results: For the purpose of validation of the proposed algorithm MATLAB Simulators is used with defined model. A sample of some 50 patients is considered and against each patient the value of blood pressure, temperature and heartbeat is saved from below and above normal values. This is defined based on the situation of emergency when the heart attach happen in real time. The deployed system is capable to process the data at its own and if it fines some uneven reading and the frequency of the measured is increased to monitoring the patient carefully.

Figure 4 shows the patient has continually high blood pressure and has the sign of heart problem and the system generate the alert and it is assessed at the concerned department. After assessment the patient is transported to hospital for further assessment and treatment.

Naive Bayes: Naive Bayes is a classification techniques based on the probability theory to find out most likely significant possible classifications.

Decision tree: This classifier uses gain ratio as an attribute selection to build a decision tree and handles the missing attributes value. It splits the values into two partitions of selected threshold value, consider the value below threshold as one child and above as another child (Table 1).

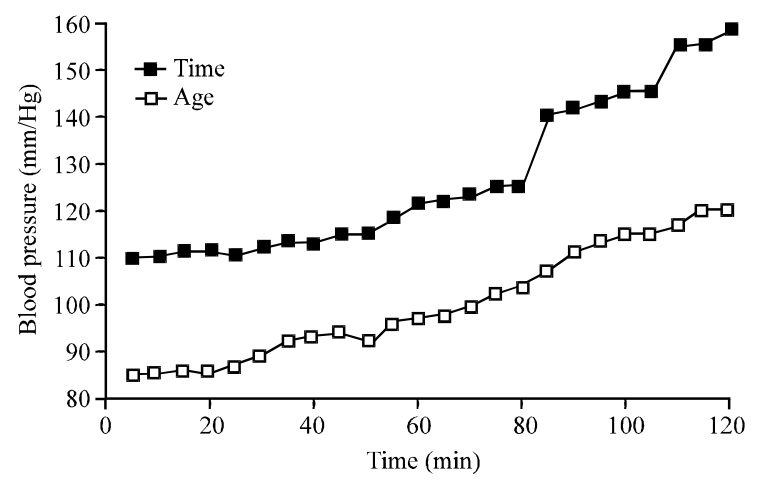

Fig. 4: Simulated results of a selected patient

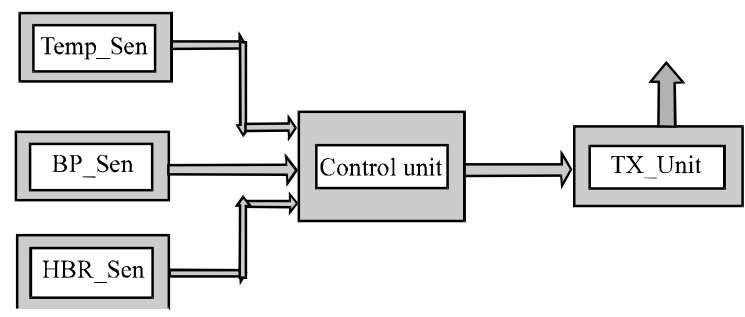

Fig. 5: Patient unit with transmitter

Table 1: Comparison between accuracies of different techniques

\begin{tabular}{lc}
\hline Techniques & Accuracy $(\%)$ \\
\hline Naive Bayes & 78.563 \\
Decision tree & 75.738 \\
Neural network & 84.449 \\
Kernel density & 82.773 \\
Naive Bayes & 95.000 \\
Decision tree & 94.930 \\
Neural network & 93.540 \\
Naive Bayes & 62.030 \\
Decision tree & 60.400 \\
Naive Bayes & 52.330 \\
KNN & 45.670 \\
Decision list & 52.000 \\
Naive Bayes & 84.140 \\
One dependency augmented Naive Bayes classifier & 80.460 \\
Genetic with decision tree & 99.200 \\
Genetic with Naive Bayes & 96.500 \\
Genetic with classification via. clustering & 88.300 \\
\hline
\end{tabular}

Figure 5 shows after measurement the information is processed at controller and saved in the history of the patient. The patient unit consists of the Temperature Sensor (Temp_Sen), Blood Pressure Sensor (BP_Sen) and Heartbeat Sensor (HBR_Sen). These sensors measures the vital under the given period and if it finds some critical information form newly sensed information then it reduce the time between the measurement. Then patient is put under care by patient unit and if needed then system initiate the alert to the monitoring system as shown in Fig. 5.

If the information is critical then it sends the information to receiver unit and that is processed at the controller. A decision is made at the controller based on 


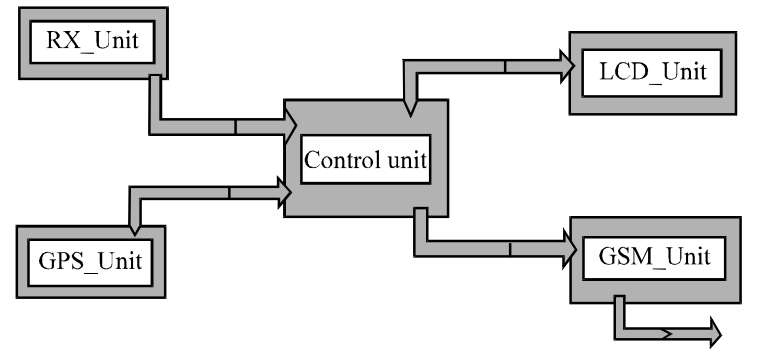

Fig. 6: Patient unit with receiver and controller

patient history to initiate emergency to the concerned department and sending the information, the system send the location of the patient by using GPS module as shown in Fig. 6.

When the rescue team reach at the location and give the 1 st aid on the scene and officer will assess the patient condition, either need to transfer to hospital or not.

As defined up that a sample is used to perform the experimentation by using MATLAB and proposed algorithm is tested theoretically, the results are very successful and reliable. The sensors sense the values (BP, Temp., HB) and communicate with controller where is it is compared with the normal and critical value saved against the patient. If the measurements are within normal range then it is saved and no action is taken but if it is falls outside the normal then the measurement frequency is increased. Then the patient put is under observation for next few hours and if the next measurement is also abnormal and alert is generated. Then it is assessed against the history of the patient, if the patient have heart disease history then it put under critical care until next measurement. If the measured data is coming continuing outside the normal range then by the GSM module send the message to concerned department. The patient location is also shared by GPS module to guide the rescue team to reach the location. At the scene rescue team assess the condition of the based on the history and current situation and if needed then transported to the hospital for further treatment.

\section{CONCLUSION}

In this research work the focus of study to monitor and track the patient for rescues purposes in case severe emergency. The proposed system is capable to acquire vitals of the patient under monitoring and process at patient unit to predict the heart attach well before the time based on the patient history. In case of uneven data coming from the vital of the patient, the system generate the alert and start monitoring the patient with defined regular intervals. In case of heart patient, initial alert message is generated to alert the rescue department. If there is continuity in the uneven data then it initiate call to the emergency number and send the current location of the patient to rescue for treatment. The designed system is assessed by using MATLAB Simulation and the results are satisfactory.

\section{RECOMMENDATIONS}

The first direction of enhancement of this reseach is to implement the proposed algorithm by using prototype in next step. The system will be designed by using ZigBee sensors to extract patient vitals and processed at controller to predict heart conditions. GPS modules will be used for location tracking and GSM module will be very used to call and generate emergency messages and calls.

\section{REFERENCES}

Alumona, T.L., V.E. Idigo and K.P. Nnoli, 2014. Remote monitoring of patients health using Wireless Sensor Networks (WSNs). IPASJ. Intl. J. Electron. Commun., 2: 90-95.

Cressie, N.A.C., 1993. Statistics for Spatial Data. 2nd Edn., John Wiley \& Sons, Hoboken, New Jersey, USA., ISBN:9780471002550, Pages: 900.

Hodgetts, T.J. and K. Mackaway, 1995. Major Incident Medical Management and Support: The Practical Approach. BMJ Publishing Group, London, England, UK.,.

Jamaluddin, M.F., 2008. Wireless heart rate monitor. Master Thesis, University of Technology Malaysia, Johor Bahru, Malaysia.

Lai, C.C., R.G. Lee, C.C. Hsiao, H.S. Liu and C.C. Chen, 2009. A H-QoS-demand personalized home physiological monitoring system over a wireless multi-hop relay network for mobile home healthcare applications. J. Netw. Comput. Appl., 32: 1229-1241.

Othman, M.F., 2006. Developing a Heartbeat Monitoring System using PIC Microcontroller. Universiti Tun Hussein Onn Malaysia, Parit Raja, Malaysia, Pages: 53.

Subudhi, S.K. and S. Sivanandam, 2014. Intelligent wireless patient monitoring and tracking system (using sensor network and wireless communication). Intl. J. Interdiscip. Multidiscip. Stud., 1: 97-104.

Super, G., S. Groth and R. Hook, 1994. Start: Simple Triage and Rapid Treatment Plan. Hoag Memorial Hospital Presbyterian, Newport Beach, California,.

Varshney, U., 2005. Pervasive healthcare: Applications, challenges and wireless solutions. Commun. Assoc. Inform. Syst., Vol. 16.

Vijendra, S., 2011. Efficient clustering for high dimensional data: Subspace based clustering and density based clustering. Inform. Technol. J., 10: 1092-1105. 